\title{
SYNTHESIS OF 7-PHENOXYPHENOTHIAZINES BY SMILES REARRANGEMENT
}

\author{
Mahmoud A. Al-abdalla, Mukesh Jain and R.R. Gupta*
}

Department of Chemistry, University of Rajasthan, Jaipur-302004, India.

\begin{abstract}
Synthesis of title compounds by Smiles rearrangement has been reported. 2-Amino-5-phenoxybenzenethiol was condensed with o-halonitrobenzenes to prepare diphenyl sulphides, which on formylation with $90 \%$ formic acid and subsequent treatment with alcoholic potassium hydroxide underwent Smiles rearrangement yielding 7-phenoxyphenothiazines. Nitrophenothiazines have been prepared by the condensation of 2-amino-5-phenoxybenzenethiol with halonitrobenzenes containing a nitro group or one nitro group and other halogen atom at both ortho positions to the reactive halogen atom as Smiles rearrangement occurs in situ.
\end{abstract}

\section{Introduction}

Phenothiazines are well known for their pharmacological and biological activities. and their several derivatives are in clinical use $(1,2)$. A slight alteration in the substitution pattern in phenothiazine nucleus causes a marked difference in the biological activities. Recently a great interest has arisen to synthesize phenothiazines to screen anticancer activities (3-12). Therefore it is considered worthwhile to synthesize unknown phenothiazines in search of better medicinal agent.

\section{Results and Discussion}

7-Phenoxyphenothiazines 6a-g have been prepared by Smiles rearrangement of 5-phenoxy-2-formamido-2-nitrodiphenyl sulphides $\underline{5}$ in alcoholic potassium hydroxide solution. The formyl derivatives were prepared by the formylation of resultant diphenyl sulphides $\underline{4}$ obtained by the condensation of 2-amino-5-phenoxybenzenethiol $\underline{1}$ with 0 -halonitrobenzenes $\underline{2}$ in ethanolic sodium acetate solution (Scheme 1).

2-Amino-5-phenoxybenzenethiol 1 required in the synthesis of title compounds has been prepared by the alkaline hydrolytic cleavage of 2-amino-6-phenoxybenzothiazole adopting the method reported elsewhere $(13,14)$.

Nitrophenothiazines have been prepared by the condensation of 2-amino-5phenoxybenzenethiol $\underline{1}$ with o-halonitrobenzenes $\underline{3}$ containing nitro group or one nitro and 
<smiles>COc1ccc(N)c(S)c1</smiles>

1<smiles>[R]c1ccc(Cl)c([N+](=O)[O-])c1</smiles>

$\underline{2}$<smiles>CC(=O)OCCOC(=O)O</smiles><smiles>[R]c1ccc(Sc2cc(OC(C)(C)C)ccc2N)c([N+](=O)[O-])c1</smiles>

$\underline{4}$

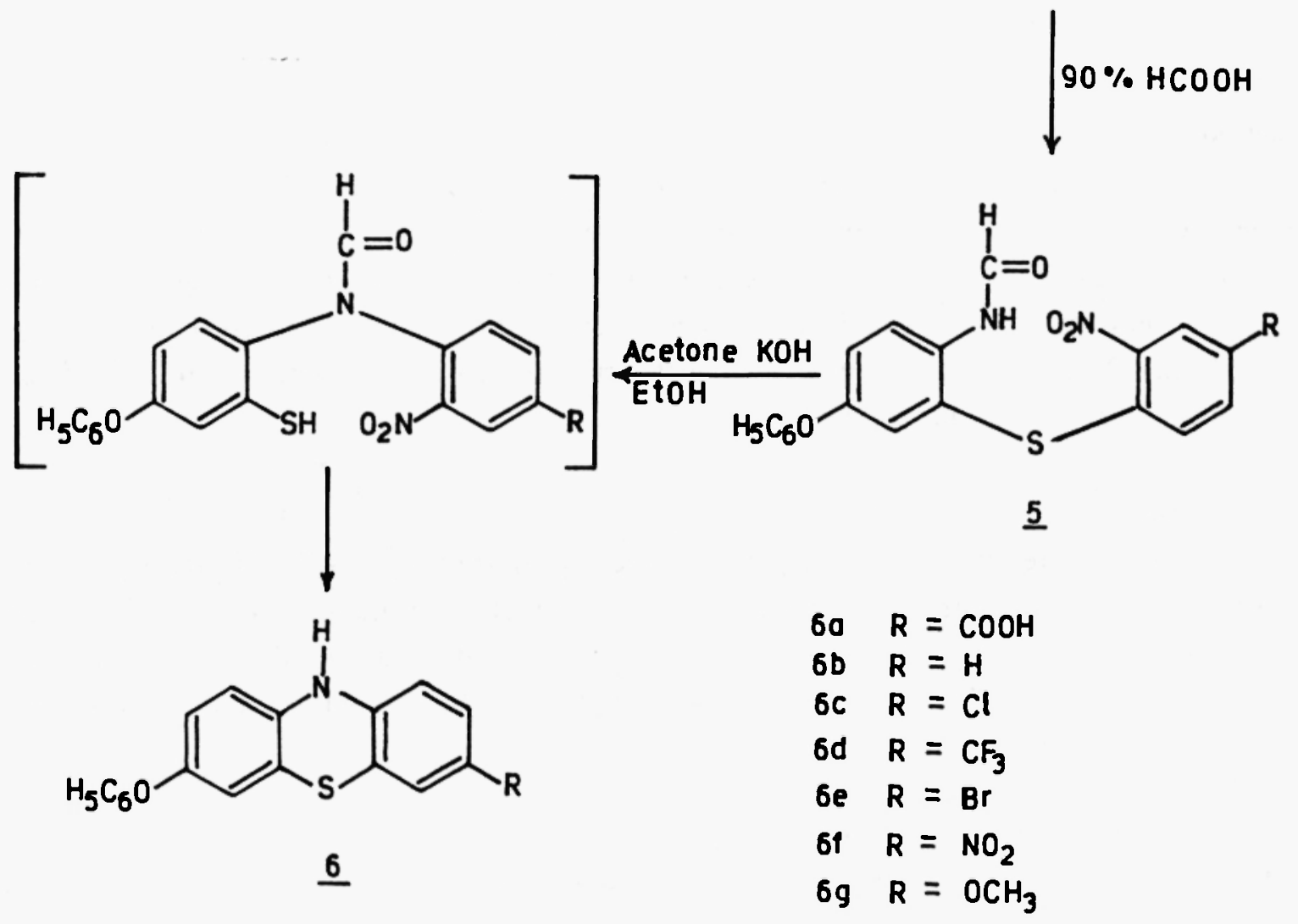

Scheme 1.

a halogen atom at both ortho positions to the reactive halogen atom in ethanolic sodium hydroxide solution where Smiles rearrangement occurs in situ (Scheme 2).

The IR spectra of phenothiazines $\underline{6 a-g}$ and $\underline{7 d}$ exhibit a sharp peak in the region $3275-3360$ $\mathrm{cm}^{-1}$ due to $\mathrm{N}-\mathrm{H}$ stretching vibrations, but phenothiazines $\underline{7 \mathrm{a}-\mathrm{c}}$ (having a nitro group at 1-position) show a large shift in the region $3275-3295 \mathrm{~cm}^{-1}$ in the secondary $\mathrm{N}-\mathrm{H}$ vibrational frequency. This shifting to lower frequency suggests a six-membered chelate 


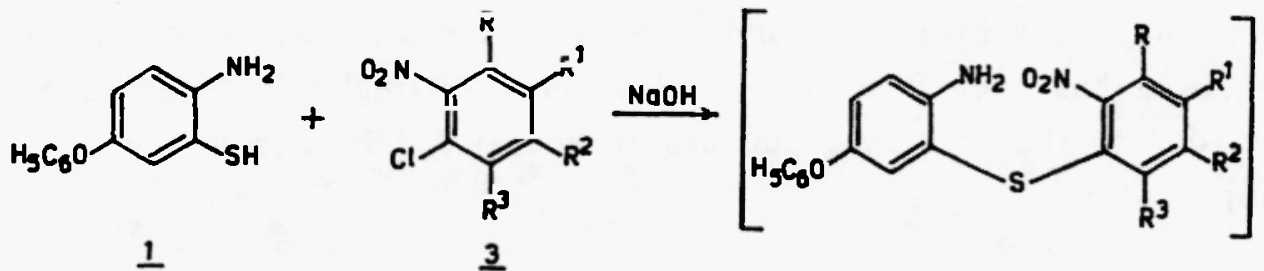

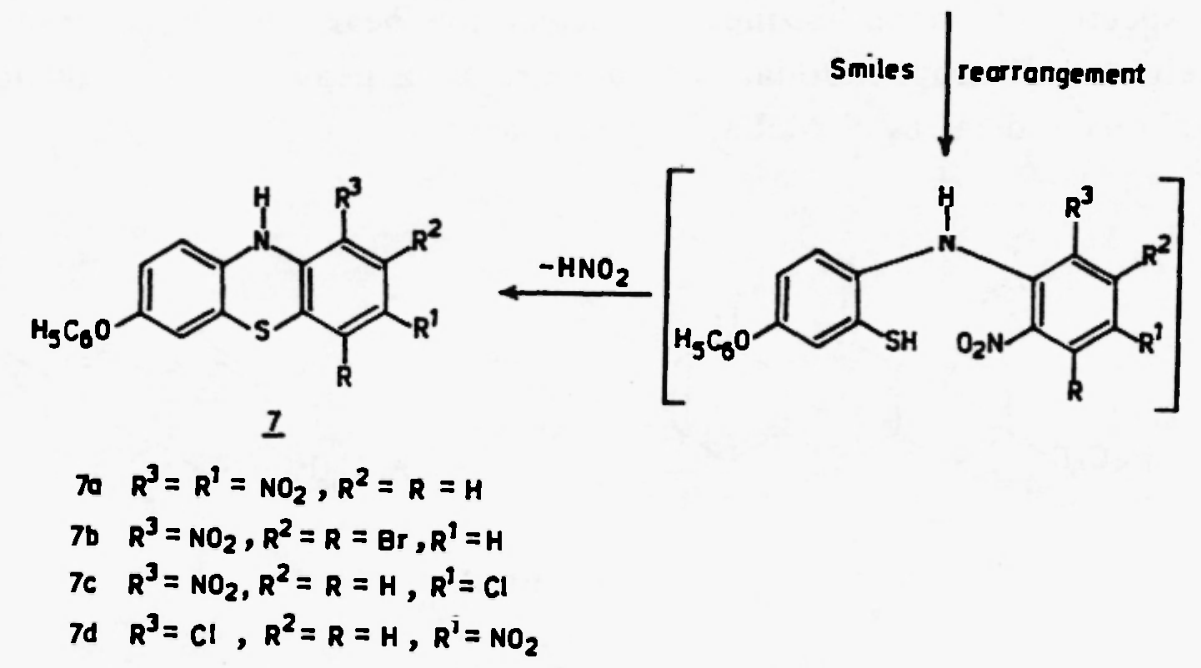

s cheme 2.

through a strong $\mathrm{NH}---\mathrm{O}=\mathrm{N}$ bonding (Fig. 1).<smiles>CCCCOc1ccc2c(c1)Sc1cccc3c1N2CON3[O-]</smiles>

Fig.1

Nitrophenothiazines $\underline{7 a-d}$ and 3-nitrophenothiazine $\underline{6 f}$ exhibit two peaks of medium intensity in the region $1550-1580 \mathrm{~cm}^{-1}$ and $1330-1385 \mathrm{~cm}^{-1}$ due to asymmetric and symmetric vibrations of the aromatic nitro group. Peaks corresponding to the chlorine atom have been observed in phenothiazines $\underline{6 c}, \underline{7 c}$ and $\underline{7 d}$ in the range $710-760 \mathrm{~cm}^{-1}$. All synthesized phenothiazines exhibit sharp peaks in the region $1215-1230 \mathrm{~cm}^{1}$ and $1005-1030 \mathrm{~cm}^{-1}$ due to $\mathrm{C}-\mathrm{O}-\mathrm{C}$ asymmetric and symmetric vibrations. Compound $\underline{6 \mathrm{~d}}$ exhibits two peaks at 1320 $\mathrm{cm}^{-1}$ and $1125 \mathrm{~cm}^{-1}$ due to $\mathrm{C}-\mathrm{F}$ stretching vibrations.

${ }^{1} \mathrm{H}$ NMR Spectra of all synthesized phenothiazines exhibit a multiplet in the region $\delta 6.41-8.68$ due to aromatic ring protons. All phenothiazines except those having a 
nitro group at 1-position show a singlet at $\delta 8.46-9.74$ due to $\mathrm{NH}$ proton. In 1-nitrophenothiazines $\underline{7 \mathrm{a}-\mathrm{c}}$, NH proton gives rise to a singlet at $\delta$ 9.81-10.30 and this downfield shift suggests a hydrogen bonding between the nitro and secondary amino group as $\mathrm{NH}---\mathrm{O}=\mathrm{N}$, as has been observed in IR spectra. A singlet is observed at $\delta 10.41$ in compound $6 \mathrm{a}$, due to the carboxy group proton and at $\delta 3.91$ in compound $6 \mathrm{~g}$ due to the methoxy group protons.

In the mass spectra of phenothiazines, molecular ion peaks are in accordance with their molecular weights. 1-Nitrophenothiazines $\underline{7 a-c}$ exhibit a peak at $\mathrm{M}^{+}-17$ which is assigned to the loss of $\mathrm{OH}^{\circ}$ radical by a McLafferty rearrangement (15) (Fig. 2).

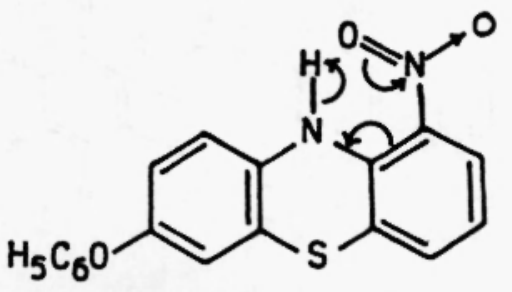

\section{Experimental}

All melting points are uncorrected. The purity of the compounds prepared has been checked by TLC. IR Spectra were recorded on a Perkin-Elmer spectrophotometer model 577 using $\mathrm{KBr}$ disc. NMR Spectra have been recorded at $90 \mathrm{MHz}$ on a Jeol FX 90Q FT NMR using TMS as an internal standard in DMSO- $d_{6}$. Mass spectra were recorded on a Jeol JMSD-300 mass spectrometer at $70 \mathrm{eV}$ with $100 \mu \mathrm{amp}$ ionising current.

\section{Preparation of 2-amino-5-phenoxy-2-nitrodiphenyl sulphides $4 \mathrm{a}-\mathrm{g}$}

To a refluxing solution of 2-amino-5-phenoxybenzenethiol $(1 ; 0.01$ mole in ethanol $20 \mathrm{ml})$ and anhydrous sodium acetate $(0.01$ mole in $5 \mathrm{ml}$ ethanol) was added an alcoholic solution of halonitrobenzene $(2 ; 0.01$ mole in ethanol $12 \mathrm{ml})$ and refluxed for three hours. The reaction mixture was concentrated and cooled overnight in an ice chamber. The solid separated out was filtered and washed with $30 \%$ ethanol. Crystallization from methanol afforded the desired product. Physical data are recorded in Table 1.

\section{Preparation of 2-formamido-5-phenoxy-2-nitrodiphenyl sulphides 5a-g}

The diphenyl sulphide obtained $(4 ; 0.01$ mole) was refluxed for three hours in $90 \%$ formic acid $(20 \mathrm{ml})$. The contents of the reaction flask were poured into a crushed ice. The solid separated out was collected, washed with water until free from acid and crystallized from benzene. Physical data are summarized in Table 1. 
Table 1 : Physical data of compound 4-7

\begin{tabular}{|c|c|c|c|c|c|c|}
\hline \multirow[t]{2}{*}{ Compound } & \multirow{2}{*}{$\begin{array}{l}\text { M.P. } \\
{\left[{ }^{\circ} \mathrm{C}\right]}\end{array}$} & \multirow{2}{*}{$\begin{array}{l}\text { Yield } \\
{[\%]}\end{array}$} & \multirow{2}{*}{$\begin{array}{c}\text { Moleculer } \\
\text { Formula }\end{array}$} & \multicolumn{3}{|c|}{ \% Found/Calcd. } \\
\hline & & & & C & $\mathbf{H}$ & $\mathbf{N}$ \\
\hline 4a & 238 & 86 & $\mathrm{C}_{19} \mathrm{H}_{14} \mathrm{~N}_{2} \mathrm{O}_{5} \mathrm{~S}$ & $\begin{array}{r}69.94 \\
6967\end{array}$ & $\begin{array}{l}3.68 \\
369\end{array}$ & $\begin{array}{l}7.34 \\
732\end{array}$ \\
\hline$\underline{4 b}$ & 113 & 94 & $\mathrm{C}_{18} \mathrm{H}_{14} \mathrm{~N}_{2} \mathrm{O}_{3} \mathrm{~S}$ & $\begin{array}{l}64.07 \\
63.03 \\
63.89\end{array}$ & $\begin{array}{l}4.17 \\
4.16\end{array}$ & $\begin{array}{l}8.24 \\
8.27\end{array}$ \\
\hline$\underline{4 c}$ & 49 & 81 & $\mathrm{C}_{18} \mathrm{H}_{13} \mathrm{ClN}_{2} \mathrm{O}_{3} \mathrm{~S}$ & $\begin{array}{l}58.12 \\
57.98\end{array}$ & $\begin{array}{l}3.52 \\
3.51\end{array}$ & $\begin{array}{l}7.48 \\
7.51\end{array}$ \\
\hline$\underline{4 d}$ & 78 & 87 & $\mathrm{C}_{19} \mathrm{H}_{13} \mathrm{~F}_{3} \mathrm{~N}_{2} \mathrm{O}_{3} \mathrm{~S}$ & $\begin{array}{l}56.27 \\
56.15\end{array}$ & $\begin{array}{l}3.23 \\
3.22\end{array}$ & $\begin{array}{l}6.87 \\
6.89\end{array}$ \\
\hline$\underline{4 e}$ & 64 & 93 & $\mathrm{C}_{18} \mathrm{H}_{13} \mathrm{BrN}_{2} \mathrm{O}_{3} \mathrm{~S}$ & $\begin{array}{l}51.69 \\
51.81\end{array}$ & $\begin{array}{l}3.14 \\
3.13\end{array}$ & $\begin{array}{l}6.69 \\
6.71\end{array}$ \\
\hline$\underline{\mathbf{4 f}}$ & 45 & 90 & $\mathrm{C}_{18} \mathrm{H}_{13} \mathrm{~N}_{3} \mathrm{O}_{5} \mathrm{~S}$ & $\begin{array}{l}56.59 \\
56.39\end{array}$ & $\begin{array}{l}3.13 \\
3.14\end{array}$ & $\begin{array}{l}10.93 \\
10.96\end{array}$ \\
\hline $4 g$ & 225 & 48 & $\mathrm{C}_{19} \mathrm{H}_{16} \mathrm{~N}_{2} \mathrm{O}_{4} \mathrm{~S}$ & $\begin{array}{l}62.21 \\
61.94\end{array}$ & $\begin{array}{l}4.38 \\
4.37\end{array}$ & $\begin{array}{l}7.63 \\
7.60\end{array}$ \\
\hline$\underline{\underline{5} \mathbf{a}}$ & 165 & 35 & $\mathrm{C}_{20} \mathrm{H}_{14} \mathrm{~N}_{2} \mathrm{O}_{6} \mathrm{~S}$ & $\begin{array}{l}58.81 \\
58.53\end{array}$ & $\begin{array}{l}3.42 \\
3.43\end{array}$ & $\begin{array}{l}6.79 \\
6.82\end{array}$ \\
\hline$\underline{5 b}$ & 199 & 39 & $\mathrm{C}_{19} \mathrm{H}_{14} \mathrm{~N}_{2} \mathrm{O}_{4} \mathrm{~S}$ & $\begin{array}{l}62.52 \\
62.28\end{array}$ & $\begin{array}{l}3.86 \\
3.85\end{array}$ & $\begin{array}{l}7.62 \\
7.64\end{array}$ \\
\hline$\underline{5 c}$ & 188 & 24 & $\mathrm{C}_{19} \mathrm{H}_{13} \mathrm{ClN}_{2} \mathrm{O}_{4} \mathrm{~S}$ & $\begin{array}{l}56.77 \\
56.93\end{array}$ & $\begin{array}{l}3.25 \\
3.24\end{array}$ & $\begin{array}{l}6.97 \\
6.99\end{array}$ \\
\hline$\underline{5 d}$ & 146 & 26 & $\mathrm{C}_{20} \mathrm{H}_{13} \mathrm{~F}_{3} \mathrm{~N}_{2} \mathrm{O}_{4} \mathrm{~S}$ & $\begin{array}{l}55.48 \\
55.30\end{array}$ & $\begin{array}{l}2.98 \\
2.99\end{array}$ & $\begin{array}{l}6.42 \\
6.45\end{array}$ \\
\hline$\underline{5 e}$ & 115 & 36 & $\mathrm{C}_{19} \mathrm{H}_{13} \mathrm{BrN}_{2} \mathrm{O}_{4} \mathrm{~S}$ & $\begin{array}{l}51.49 \\
51.24\end{array}$ & $\begin{array}{l}2.95 \\
2.94\end{array}$ & $\begin{array}{l}6.31 \\
6.29\end{array}$ \\
\hline$\underline{5 f}$ & 186 & 38 & $\mathrm{C}_{19} \mathrm{H}_{13} \mathrm{~N}_{3} \mathrm{O}_{6} \mathrm{~S}$ & $\begin{array}{l}55.64 \\
55.47\end{array}$ & $\begin{array}{l}3.17 \\
3.18\end{array}$ & $\begin{array}{l}10.24 \\
10.21\end{array}$ \\
\hline $5 \mathrm{~g}$ & 182 & 32 & $\mathrm{C}_{20} \mathrm{H}_{16} \mathrm{~N}_{2} \mathrm{O}_{5} \mathrm{~S}$ & $\begin{array}{l}60.70 \\
60.59\end{array}$ & $\begin{array}{l}4.07 \\
4.06\end{array}$ & $\begin{array}{l}7.04 \\
7.06\end{array}$ \\
\hline$\underline{6 a}$ & 276 & 52 & $\mathrm{C}_{19} \mathrm{H}_{13} \mathrm{NO}_{3} \mathrm{~S}$ & $\begin{array}{l}68.15 \\
68.04\end{array}$ & $\begin{array}{l}3.91 \\
3.90\end{array}$ & $\begin{array}{l}4.18 \\
4.17\end{array}$ \\
\hline$\underline{6 b}$ & 61 & 63 & $\mathrm{C}_{18} \mathrm{H}_{13} \mathrm{NOS}$ & $\begin{array}{l}74.41 \\
74.20\end{array}$ & $\begin{array}{l}4.48 \\
4.49\end{array}$ & $\begin{array}{l}4.82 \\
4.80\end{array}$ \\
\hline$\underline{6 c}$ & 122 & 27 & $\mathrm{C}_{18} \mathrm{H}_{12} \mathrm{ClNOS}$ & $\begin{array}{l}66.21 \\
66.35\end{array}$ & $\begin{array}{l}3.69 \\
3.71\end{array}$ & $\begin{array}{l}4.27 \\
4.29\end{array}$ \\
\hline$\underline{6 \mathrm{~d}}$ & 65 & 57 & $\mathrm{C}_{19} \mathrm{H}_{12} \mathrm{~F}_{3} \mathrm{NOS}$ & $\begin{array}{l}63.30 \\
63.50\end{array}$ & $\begin{array}{l}3.35 \\
3.36\end{array}$ & $\begin{array}{l}3.88 \\
3.89\end{array}$ \\
\hline$\underline{6 e}$ & 89 & 56 & $\mathrm{C}_{18} \mathrm{H}_{12} \mathrm{BrNOS}$ & $\begin{array}{l}58.14 \\
58.38\end{array}$ & $\begin{array}{l}3.25 \\
3.26\end{array}$ & $\begin{array}{l}3.79 \\
3.78\end{array}$ \\
\hline$\underline{6 f}$ & 114 & 41 & $\mathrm{C}_{18} \mathrm{H}_{12} \mathrm{~N}_{2} \mathrm{O}_{3} \mathrm{~S}$ & $\begin{array}{l}64.03 \\
64.27\end{array}$ & $\begin{array}{l}3.60 \\
3.59\end{array}$ & $\begin{array}{l}8.30 \\
8.32\end{array}$ \\
\hline $6 g$ & 57 & 31 & $\mathrm{C}_{19} \mathrm{H}_{15} \mathrm{NO}_{2} \mathrm{~S}$ & $\begin{array}{l}71.25 \\
71.00\end{array}$ & $\begin{array}{l}4.72 \\
4.70\end{array}$ & $\begin{array}{l}4.35 \\
4.35\end{array}$ \\
\hline$\underline{7 a}$ & 218 & 40 & $\mathrm{C}_{18} \mathrm{H}_{14} \mathrm{~N}_{3} \mathrm{O}_{5} \mathrm{~S}$ & $\begin{array}{l}56.43 \\
56.69\end{array}$ & $\begin{array}{l}2.91 \\
2.90\end{array}$ & $\begin{array}{l}11.04 \\
11.01\end{array}$ \\
\hline$\underline{7 b}$ & 68 & 21 & $\mathrm{C}_{18} \mathrm{H}_{10} \mathrm{Br}_{2} \mathrm{~N}_{2} \mathrm{O}_{3} \mathrm{~S}$ & $\begin{array}{l}43.64 \\
43.75\end{array}$ & $\begin{array}{l}2.03 \\
2.03\end{array}$ & $\begin{array}{l}5.65 \\
5.66\end{array}$ \\
\hline$\underline{7 c}$ & 56 & 49 & $\mathrm{C}_{18} \mathrm{H}_{11} \mathrm{ClN}_{2} \mathrm{O}_{3} \mathrm{~S}$ & $\begin{array}{l}58.17 \\
58.30\end{array}$ & $\begin{array}{l}2.99 \\
2.98\end{array}$ & $\begin{array}{l}7.53 \\
7.55\end{array}$ \\
\hline$\underline{7 d}$ & 49 & 48 & $\mathrm{C}_{18} \mathrm{H}_{11} \mathrm{ClN}_{2} \mathrm{O}_{3} \mathrm{~S}$ & $\begin{array}{l}58.45 \\
58.30 \\
\end{array}$ & $\begin{array}{l}2.97 \\
2.98 \\
\end{array}$ & $\begin{array}{l}7.57 \\
7.55 \\
\end{array}$ \\
\hline
\end{tabular}




\section{Preparation of 7-phenoxyphenothiazines 6a-g}

To a refluxing solution of formyl derivatives $(\underline{5} ; 0.01 \mathrm{~mole})$ in acetone $(15 \mathrm{ml})$ was added an alcoholic solution of potassium hydroxide $(0.2 \mathrm{gm}$ in $5 \mathrm{ml}$ ethanol). The colour of the solution is darkened immediately on addition of the alkaline alcoholic solution. The contents were heated for 30 mins. To this solution a second lot of potassium hydroxide (0.2 $\mathrm{gm}$ in $5 \mathrm{ml}$ ethanol) was added and refluxing was continued for two hours. The contents were cooled down and poured into a beaker containing crushed ice. The solid separated out was filtered, washed with cold water and finally with $30 \%$ ethanol. Crystallization from methanol/benzene afforded the desired phenothiazines. The physical data are summarized in Table 1.

\section{Preparation of nitrophenothiazines $\underline{7 a-d}$}

To a stirred suspension of 2-amino-5-phenoxybenzenethiol ( $1 ; 0.01$ mole) and a reactive halonitrobenzene $(\underline{3} ; 0.01$ mole in $20 \mathrm{ml}$ ethanol) was added an alcoholic solution of sodium hydroxide $(0.01$ mole) and contents were refluxed for two hours. The reaction mixture was cooled down, filtered, washed with hot water and finally with $20 \%$ ethanol. Crystallization from methanol/acetone affords nitrophenothiazines. Physical data are summarized in Table 1.

\section{References}

1. R. R. Gupta (Ed.), " Phenothiazines and 1,4-Benzothiazines - Chemical and Biomedical Aspects", Elsevier, Amsterdam (1988).

2. H. Keyzer, G. M. Eckert, I. S. Forrest, R. R. Gupta, F. Gutmamn, J. Molnar (Eds.), "Thiazines and Structurally Related Compounds", (Proceedings of Sixth International Conference on Phenothiazines and Structurally Related Psychotropic Compounds) Pasadena, California Sept. 11-14 (1990), Krieger Publishing Co., Malabar, Florida, USA (1992).

3. R. R. Gupta, M. Jain, R. S. Rathore and A. Gupta, J. Fluor. Chem. 62, 191 (1993).

4. A. Andreani, M. Rambaldi, A. Locatelli, P. Aresca, R. Bossa and I. Galatulas, Eur. J. Med, Chem., 26,113 (1991).

5. N. Motohashi, S. R. Gollapudi, J. Emrani and K. R. Battiprolu, Canc. Invest., 2(3), 305 (1991).

6. N. Motohashi, Anticanc. Res., 11, 1125 (1991).

7. R. Ganapathi and D. Grabowski, Canc. Res., 43, 3693 (1983).

8. N. Motohashi, Yakagaku Zasshi. 103, 364 (1983); Chem. Abstr., 29, 231 (1983).

9. Daicel Co. Ltd., Jap. Kokai Koho Jp., 57, 175, 181 (1982); Chem. Abstr., 28, 160729 (1983).

10. K. K. Showa, Jap. Kakai Tokkyo Koho Jp., 81, 166, 182 (1982); Chem. Abstr., 26, 142871 (1982).

11. Daicel Chemical Industries Ltd., Jap. kokai Tokkyo Koho Jp., 57, 185, 271 (1982); Chem. Abstr., 28, 160730 (1983).

12. V. A. Rigas, H. V. Vunakis, L. Levine, Prostaglandins Med., Z(2), 183 (1981); Chem. Abstr., 25 , 197206 (1981).

13. R. R. Gupta, K. G. Ojha, G. S. Kalwania and M. Kumar, Heterocycles 14, 1145 (1980).

14. R. R. Gupta, K. G. Ojha and M. Kumar, J. Heterocycl. Chem. 17. 1325 (1980).

15. F. W. McLafferty, Anal. Chem. 31, 82 (1959).

Received May 10, 1995 